\title{
An efficient algorithm for exact computation of system and survival signatures using binary decision diagrams
}

\section{Sean Reed}

Resilience Engineering Research Group, University of Nottingham, University Park, Nottingham NG7 2RD, UK

\begin{abstract}
System and survival signatures are important and popular tools for studying and analysing the reliability of systems. However, it is difficult to compute these signatures for systems with complex reliability structure functions and large numbers of components. This paper presents a new algorithm that is able to compute exact signatures for systems that are far more complex than is feasible using existing approaches. This is based on the use of reduced order binary decision diagrams (ROBDDs), multidimensional arrays and the dynamic programming paradigm. Results comparing the computational efficiency of deriving signatures for some example systems (including complex benchmark systems from the literature) using the new algorithm and a comparison enumerative algorithm are presented and demonstrate a significant reduction in computation time and improvement in scalability with increasing system complexity.
\end{abstract}

\section{Keywords}

system signature, survival signature, binary decision diagram, system reliability

\section{Notation}

$m \quad$ Number of components in the system

$K \quad$ Number of component types in the system, where components of the same type have exchangeable random failure times

$M_{k} \quad$ Number of components of type $k$

$x_{i} \quad$ Boolean variable representing the state of component $i$ where $x_{i}=1$ if the component functions and $x_{i}=0$ if the component is failed

Vector of length $m$ representing the system component states where the value at index $i \in\{1, \ldots, m\}$ corresponds to $x_{i}$

$\phi \quad$ Boolean function representing the system reliability structure where $\phi(x)=1$ if the system functions with component state vector $x$ and $\phi(x)=0$ if the system is failed

$f_{x_{i}=v} \quad$ Boolean function $f$ evaluated with Boolean variable $x_{i}=v$

$T_{s} \quad$ Random failure time of the system

$T_{l: m} \quad$ lth order statistic for the random component failure times

$q_{l} \quad$ Probability that the system failure time coincides with the lth component failure (i.e. $\left.P\left(T_{s}=T_{l: m}\right)\right)$

$q \quad$ Vector of length $m$ known as the system signature where the value at index $l \in\{1, \ldots, m\}$ corresponds to $q_{j}$ 
$S_{l_{1}, \ldots, l_{K}} \quad$ Set of state vectors for the $m$ components that contain precisely $l_{k}$ functioning components of type $k$

$\left|S_{l_{1}, \ldots, l_{K}}\right| \quad$ Cardinality of $S_{l_{1}, \ldots, l_{K}}$

$S \quad$ Multidimensional array with $K$ dimensions where the value at index $\left(l_{1}, \ldots, l_{K}\right)$ in dimensions $(1, \ldots, K)$ respectively corresponds to $S_{l_{1}, \ldots, l_{K}}$

$|S| \quad$ Multidimensional array with $K$ dimensions where the value at index $\left(l_{1}, \ldots, l_{K}\right)$ in dimensions $(1, \ldots, K)$ respectively corresponds to $\left|S_{l_{1}, \ldots, l_{K}}\right|$

$\bar{\Phi}_{l_{1}, \ldots, l_{K}} \quad$ Number of state vectors for the $m$ components that both contain precisely $l_{k}$ functioning components of type $k$ and result in the system functioning

$\bar{\Phi} \quad$ Multidimensional array with $K$ dimensions where the value at index $\left(l_{1}, \ldots, l_{K}\right)$ in dimensions $(1, \ldots, K)$ respectively corresponds to $\bar{\Phi}_{l_{1}, \ldots, l_{K}}$

$\Phi_{l_{1}, \ldots, l_{K}}$ Probability that the system functions given that exactly $\left(l_{1}, \ldots, l_{K}\right)$ components of types $(1, \ldots, K)$ respectively function

$\Phi \quad$ Multidimensional array with $K$ dimensions known as the survival signature where the value at index $\left(l_{1}, \ldots, l_{K}\right)$ in dimensions $(1, \ldots, K)$ respectively corresponds to $\Phi_{l_{1}, \ldots, l_{K}}$

$C_{t}^{k} \quad$ Number of components of type $k$ in the system that function at time $t>0$

$F_{k} \quad$ Cumulative distribution function for the time to failure of components of type $k$

$A^{\ddagger} \quad$ The complement of multidimensional array A

$A \oplus B \quad$ Elementwise addition of multidimensional arrays $\mathrm{A}$ and $\mathrm{B}$

$A \ominus B \quad$ Elementwise subtraction of multidimensional array A from multidimensional array B

$A \oslash B \quad$ Elementwise division of multidimensional array A from multidimensional array B

$A \boxplus k \quad$ Resize-k operation on multidimensional array A

$A \circledast k \quad$ Shift-k operation on multidimensional array A

\section{Introduction}

The system signature, introduced by Samaniego [1], is a useful tool for studying the reliability of coherent systems [2]. Consider a coherent system of $m$ components with independent identically distributed failure times. Let $T_{s}>0$ be the random failure time of the system and let $T_{j: m}$ be the $l$ th order statistic for the random component failure times with $T_{1: m} \leq T_{2: m} \leq \cdots \leq T_{m: m}$. The system signature is defined as the vector $q$ where the value at index $l \in\{1,2, \ldots, m\}$, denoted $q_{l}$, gives the probability that the system failure time coincides with the lth component failure

$$
q_{l}=P\left(T_{s}=T_{l: m}\right)
$$

The system signature has various theoretical applications in reliability engineering such as establishing stochastic comparisons between the reliability of different systems [3,4]. An overview of the system signature and some of its applications in reliability engineering is given by Samaniego [2], whilst Eryilmaz [5] gives a review of recent advances. Recently, Coolen and Coolen-Maturi [6] introduced the survival signature which, 
similar to the system signature, fulfils the role of a quantitative model of the system reliability structure that is entirely separated from the random failure times of the components. The survival signature has the advantage that is can be easily generalised to systems with multiple types of components unlike the system signature for which this is practically impossible [6]. This generalisation represents a significant practical advantage since many systems contain multiple component types, including networks which contain at least two types of component ('nodes' and 'links'). Let $x=\left(x_{1}, x_{2}, \ldots, x_{m}\right) \in\{0,1\}^{m}$ represent a Boolean state vector for a system of $m$ components with exchangeable failure times, where $x_{i}=1$ if component $i$ functions and $x_{i}=0$ if it is failed. Also let $\phi:\{0,1\}^{m} \rightarrow\{0,1\}$ represent the system reliability structure function, defined for all $2^{m}$ possible $x$, where $\phi(x)=1$ if the system functions with component states $x$ and $\phi(x)=0$ if it is failed. Finally, let $S_{l}$ denote the set of component state vectors with exactly $l$ of the $m$ components functioning (i.e. $\sum_{i=1}^{m} x_{i}=l$ ). The survival signature is then defined as the vector $\Phi$ where the value at index $l \in$ $\{0,1,2, \ldots, m\}$, denoted $\Phi_{l}$, gives the probability that the system functions given that precisely $l$ components function

$$
\Phi_{l}=\left(\begin{array}{c}
m \\
l
\end{array}\right)^{-1} \sum_{x \in S_{l}} \phi(x)
$$

Now consider the case where the $m$ components in the system are partitioned into $K$ different types, where the $M_{k}$ components of type $k \in\{1, \ldots, K\}$ have exchangeable random failure times. Let $S_{l_{1}, \ldots, l_{K}}$ denote the set of component state vectors that contain precisely $l_{k} \in\left\{0,1, \ldots, M_{k}\right\}$ functioning components of type $k$ (i.e. those for which $\sum_{i=1}^{M_{k}} x_{i}^{k}=l_{k}$ for $k=0,1, \ldots, K-1$ where $x_{i}^{k}$ is the ith component of type $k$ ). Also let $\left|S_{l_{1}, \ldots, l_{K}}\right|=\prod_{k=1}^{K}\left(\begin{array}{c}M_{k} \\ l_{k}\end{array}\right)$ denote the cardinality of $S_{l_{1}, \ldots, l_{K}}$ and $\bar{\Phi}_{l_{1}, \ldots, l_{K}}=\sum_{x \in S_{l_{0}, \ldots, l_{K-1}}} \phi(x)$ denote the number of state vectors from $S_{l_{1}, \ldots, l_{K}}$ for which the system functions. The generalised survival signature, $\Phi$, is then defined as the multidimensional array with $K$ dimensions where the value at index $\left(l_{1} \in\left\{0, \ldots, M_{1}\right\}, \ldots, l_{K} \in\right.$ $\left.\left\{0, \ldots, M_{K}\right\}\right)$ in dimensions $(1, \ldots, K)$ respectively, denoted $\Phi_{l_{1}, \ldots, l_{K}}$, gives the probability that the system functions given that precisely $\left(l_{1}, \ldots, l_{K}\right)$ components of types $(1, \ldots, K)$ respectively function

$$
\Phi_{l_{1}, \ldots, l_{K}}=\frac{\bar{\Phi}_{l_{1}, \ldots, l_{K}}}{\left|S_{l_{1}, \ldots, l_{K}}\right|}
$$

Let $C_{t}^{k} \in\left\{0, \ldots, M_{k}\right\}$ denote the number of components of type $k$ in the system that function at time $t>0$. The probability that the system functions at time $t$ can be calculated using the survival signature and the joint probability distribution for the number of functioning components of each type at time $t$

$$
P\left(T_{S}>t\right)=\sum_{l_{1}=0}^{M_{1}} \ldots \sum_{l_{K}=0}^{M_{K}}\left[\Phi_{l_{1}, \ldots, l_{K}} P\left(\bigcap_{k=1}^{K}\left\{C_{t}^{k}=l_{k}\right\}\right)\right]
$$

If failure times of components of type $k$ are conditionally independent and identically distributed with CDF $F_{k}(t)$ and failure times of components of different types are independent, then

$$
P\left(T_{S}>t\right)=\sum_{l_{1}=0}^{M_{1}} \ldots \sum_{l_{K}=0}^{M_{K}}\left[\Phi_{l_{1}, \ldots, l_{K}} \prod_{k=1}^{K}\left(\left(\begin{array}{c}
M_{k} \\
l_{k}
\end{array}\right)\left[F_{k}(t)\right]^{M_{k}-l_{k}}\left[1-F_{k}(t)\right]^{l_{k}}\right)\right]
$$

For systems containing a single component type, the system signature and survival signature have the simple relation

$$
q_{l}=\Phi_{m-l}-\Phi_{m-l-1}
$$


Several theoretical applications of the survival signature to problems in the field of reliability engineering have already been published including nonparametric predictive inference for system reliability [7]; Bayesian inference for reliability of systems and networks [8]; modelling uncertain aspects of system dependability [9]; predictive inference for system reliability after common-cause component failures [10]; imprecise system reliability and component importance [11]; Bayesian nonparametric system reliability using sets of priors [12] and comparing systems with heterogeneous components [13].

Despite the advances is the theory and development of numerous application for signatures, practical applications have until now been limited to the analysis of relatively small problems. The main reason for this is that the computation of signatures using existing methods is difficult unless the number of components is small or the system reliability structure function is quite trivial $[2,6]$. The aim of this paper is to present a new and computationally efficient algorithm based on binary decision diagrams for computing exact system and survival signatures and report its computational efficiency for a number of example systems, including large and complex systems that have been derived from practice and were published as benchmarks in the literature. The remainder of this paper is organised as follows: Section 2 describes the existing methods that are available for the computation of system and survival signatures. Section 3 introduces the new algorithm. Section 4 presents some results on the efficiency of the new algorithm in computing system and survival signatures for a set of example systems, including some large and complex benchmark systems from the literature. Section 5 summarises the paper, gives some concluding remarks and also discusses limitations and areas for future work.

\section{Existing Methods for System and Survival Signature Computation}

A small number of methods for computing system signatures have been published in the literature and are based on minimal ordered cut sets, diagonal sections of the reliability structure function and generating functions. Kochar et al [14] note that the system signature can be defined for $j \in\{1,2, \ldots, m\}$ as

$$
q_{j}=\frac{\text { number of component orderings for which the jth failure causes system failure }}{m !}
$$

The system signature can therefore be computed by generating the $m$ ! permutations of component orderings and counting the number of permutations for which the $j$ th component failure results in system failure for $j \in$ $\{1,2, \ldots, m\}$. The system failure evaluation might be carried out, for example, by deriving the minimal cut sets for the system and comparing them with subsets of increasing size from each component ordering permutation until a match is found. A subset of components from a system is a cut set if the failure of those components implies failure of the system and is a minimal cut set if it has no proper subset that is also a cut set. Since the computational expense of this approach grows approximately with the product of $m$ ! and the number of minimal cut sets, it is only feasible for simple systems. Boland [15] showed that the system signature for a system could also be defined for $j \in\{1,2, \ldots, m\}$ as

$$
q_{j}=\frac{\text { number of minimal ordered cut sets of size } \mathrm{j}}{m ! \div(m-j) !}
$$

where an ordered minimal cut set is an ordered sequence of component failures that results in system failure only when the final component in the sequence fails. In this alternative expression, only permutations of $j$ of the $m$ components need to be counted for $q_{j}$, however the computational expense still increases exponentially with the number of components in the system making it infeasible when $m$ is large. Marichal and Mathonet [16] proposed an alternative to the method from Boland [15] that avoids requiring evaluation of the system reliability structure function for every permutation of component orderings, showing that the system signature 
can be computed for a system from the diagonal section of the reliability structure function via derivatives. This method relies on complicated algebraic manipulations and is best suited to hand calculation with systems with small numbers of components. Triantafyllou and Koutras [17] presented an approach for computing the system signatures for linear and circular k-out-of-n: F systems using generating functions. Linear and circular k-out-of-n: F systems consist of linear and circularly arranged components, respectively, where the system fails if and only if at least $k$ consecutive components fail. Whilst this method is computation efficient it is applicable only to the small subset of systems that can be modelled as a linear or circular k-out-of-n: F system such as certain telecommunication and pipeline networks. For systems that can be decomposed into disjoint subsystems, Marichal and Mathonet [16] and Da et al [18] derived formulas for computing the system signature of a system from the system signatures computed for its subsystems and the reliability structure function for the system in terms of its subsystems. This reduces the computational burden compared to computing the system signature for the complete system directly.

An enumerative approach can be used for the computation of the survival signature, where each possible state vector is evaluated in turn, such as the following approach used by the ReliabilityTheory package [19] for the R programming language [20]:

- Derive the minimal cut sets for the system.

- Generate each of the $2^{m}$ possible component state vectors.

- Compare each component state vector to the minimal cut sets to determine whether it results in the system functioning or not.

- Count the number of component state vectors with $\left(l_{1}, \ldots, l_{K}\right)$ components of types $(1, \ldots, K)$ functioning that result in system functioning to obtain $\bar{\Phi}_{l_{1}, \ldots, l_{K-1}}$ for all $\prod_{k=1}^{K} M_{k}+1$ different $\left(l_{1}, \ldots, l_{K}\right)$.

- Obtain $\Phi_{l_{1}, \ldots, l_{K}}$ by dividing $\bar{\Phi}_{l_{1}, \ldots, l_{K}}$ by $\left|S_{l_{1}, \ldots, l_{K}}\right|$ for all $\prod_{k=1}^{K} M_{k}+1$ different $\left(l_{1}, \ldots, l_{K}\right)$.

However, the computational expense of this approach grows approximately with the product of $2^{m}$ and the number of minimal cut sets, it becomes infeasible for complex systems (e.g. for $m=30$ there are over 1 billion possible component state vectors to consider).

In summary, the existing methods for computing system and survival signatures are only computationally feasible when applied to relatively simple systems with small numbers of components. If the system can be reduced to a set of disjoint subsystems, then the combinational formulas from Marichal and Mathonet [16] and Da et al [18] can be useful in reducing computational burden but do not address the challenge of deriving the signatures for the subsystems. The method from Triantafyllou and Koutras [17] is efficient but its applicability is limited to the small number of systems that can be modelled as linear or circular k-out-of-n: $\mathrm{F}$ systems. Therefore new methods are required to enable the practical computation of system and survival signature for many large and complex real world systems.

3 Description of New Method for System and Survival Signature Computation based on Binary Decision Diagrams

A new algorithm for efficiently computing system and survival signatures is presented in this section. The algorithm utilises reduced ordered binary decision diagrams (hereafter referred to as BDD) and multidimensional array data structures in the signature computation process.

3.1 Reduced Ordered Binary Decision Diagram (BDD) Data Structure

A BDD [21] is a data structure in the form of a rooted directed acyclic graph which can be used to compactly represent and efficiently manipulate a Boolean function. They are based upon Shannon decomposition theory [22]. The Shannon decomposition of a Boolean function $f$ on Boolean variable $x_{i}$ is defined as 


$$
f=\left(\left(x_{i}=1\right) \wedge f_{x_{i}=1}\right) \vee\left(\left(x_{i}=0\right) \wedge f_{x_{i}=0}\right)
$$

where $f_{x_{i}=v}$ is $f$ evaluated with $x_{i}=v$.

Each BDD contains two terminal nodes that represent the Boolean constant values 1 and 0 . Each non-terminal node represents a subfunction $g$, is labelled with a Boolean variable $v$ and has two outgoing edges. By applying a total ordering on the $m$ Boolean variables for function $f$ by mapping them to the integers $0, \ldots, m-1$, and applying the Shannon decomposition recursively to $f$, it can be represented as a binary tree with $m+1$ levels. Each intermediate node, referred to as an if-then-else (ite) node, at level $l \in\{0, \ldots, m-1\}$ (where the root node is at level $O$ and the nodes at level $m-1$ are adjacent to the terminal nodes) represents a Boolean function $g$ on variables $x_{l}, x_{l+1}, \ldots, x_{m-1}$. It is labelled with variable $x_{l}$ and has two out edges called 1-edge and 0 -edge linking to nodes labelled with variables higher in the ordering. 1 -edge corresponds to $x_{l}=1$ and links to the node representing $g_{x_{l}=1}$, whist 0 -edge corresponds to $x_{l}=0$ and links to the node representing $g_{x_{l}=0}$. In addition, the following two reduction rules are applied:

1. Isomorphic subgraphs are merged.

2. Any node whose two children are isomorphic is eliminated.

Complement edges [23] are an extension to standard BDDs that reduce size and computation time. A complement edge is an ordinary edge that is marked to indicate that the connected child node (at a higher level) is to be interpreted as the complement of its Boolean function. The use of complement edges is limited to the 0 -edges to ensure canonicity. In general, the BDD representation of a Boolean function of $m$ variables has far fewer nodes than $2^{m}$ nodes.

Efficient algorithms have been developed for the computation of the BDD representation of the reliability structure function of a system from fault trees [24], event trees [25], networks [26] and dynamic flowgraphs [27]. The chosen variable ordering often has a significant impact on the size of the resultant BDD. Finding the optimum variable ordering is known to be a NP-hard problem [28], however many efficient heuristic methods have been developed (e.g. for fault trees [29] or networks [30]).

An example of a success tree (i.e. logical complement of a fault tree, where basic events and the top event represent component and system functioning respectively) is shown in Figure 1a. Figure 1b shows a complemented edge BDD representation of the success tree, where components are ordered $A<B<C<D$, ite nodes are labelled with the component names, the terminal 1 node is labelled 1 , the dashed edges represent 0 -edges (marked with -1 if complemented) and solid edges represent 1-edges. 

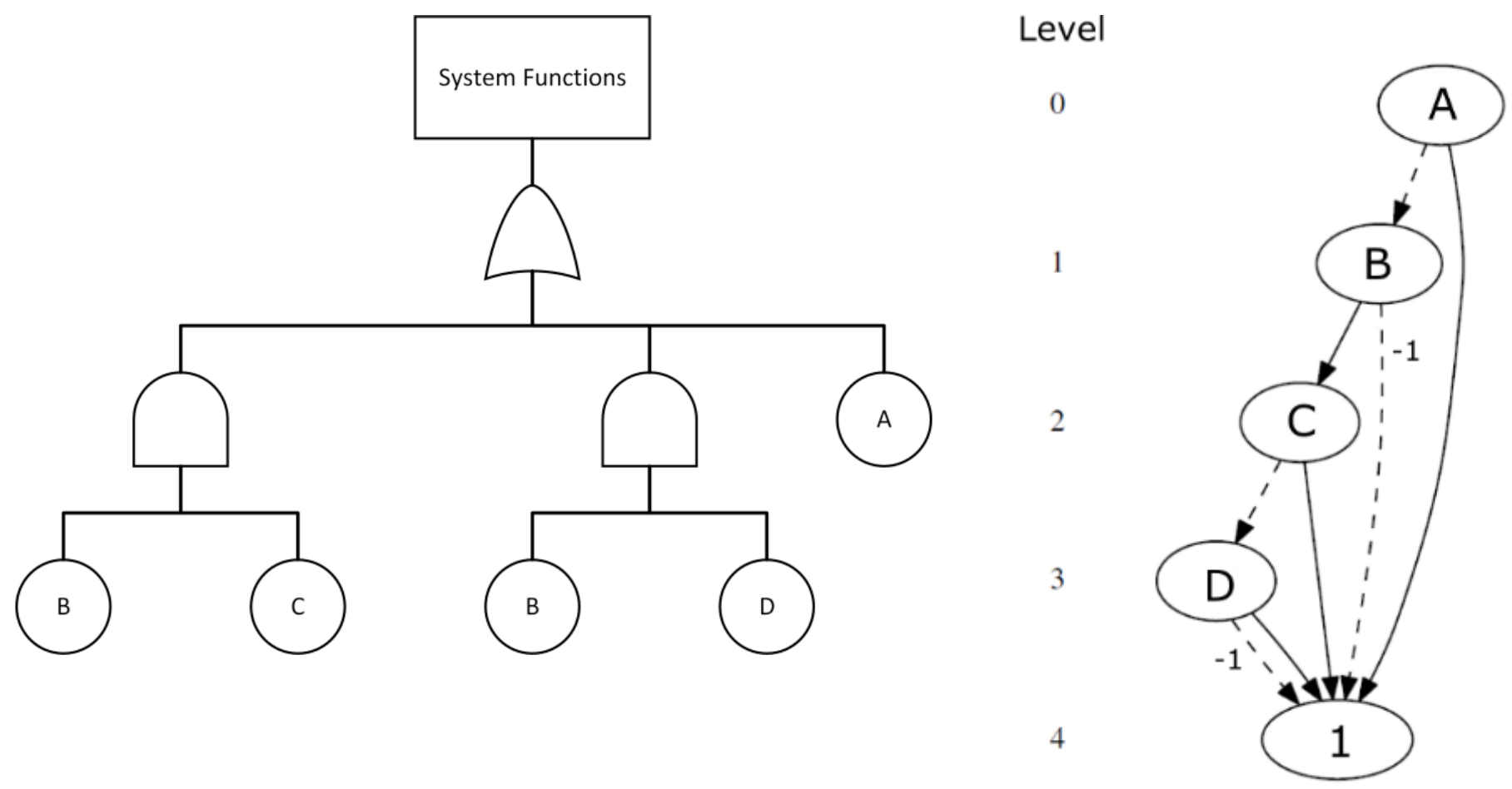

Figure 1a - A success tree from an example system.

Figure 1b - A complemented edge BDD representation of the success tree shown in Figure 1a.

\subsection{Computational representation of $\Phi$ and $\bar{\Phi}$}

The number of possible values for $\Phi_{l_{1}, \ldots, l_{K}}$ or $\bar{\Phi}_{l_{1}, \ldots, l_{K}}$ from Eqn. 3 for any $\left(l_{1}, \ldots, l_{K}\right)$, across all possible systems with $K$ component types and $M_{k}$ components of type $k$, is given by $\prod_{k=1}^{K}\left(\max \left(\left(\begin{array}{c}M_{k} \\ {\left[M_{k} \div 2\right\rceil}\end{array}\right),\left(\begin{array}{c}M_{k} \\ {\left[M_{k} \div 2\right]}\end{array}\right)\right)\right)$. Thus the range of possible values can be very large for systems with many components and component types and require high bit length numerical data structures to represent computationally to exact or high precision. Additionally there are a total of $\prod_{k=1}^{K} M_{k}+1$ different $\left(l_{1}, \ldots, l_{K}\right)$ indices for which $\Phi_{l_{1}, \ldots, l_{K}}$ and $\bar{\Phi}_{l_{1}, \ldots, l_{K}}$ from Eqn. 3 need to be computed. These values can be represented computationally using a multidimensional array data structure with $K$ dimensions and length $M_{k}+1$ in dimension $k$, where the value stored at index $\left(l_{1}, \ldots, l_{K}\right)$ of the array is equal to $\Phi_{l_{1}, \ldots, l_{K}}$ or $\bar{\Phi}_{l_{1}, \ldots, l_{K}}$. Across all possible systems with $m$ components and $K$ component types, the maximum possible value for $\prod_{k=1}^{K} M_{k}+1$ is $\left(\frac{m}{K}+1\right)^{K}$. Therefore, the computational representation of $\bar{\Phi}$ or $\Phi$ for systems with many components and component type can require significant amount of memory. For example, the computational representation of the survival signature for a 50 component system partitioned evenly across 10 component types would require the use of a multidimensional array with 60,466,176 elements and 24 bits of memory per element to ensure exact representation of $\bar{\Phi}$ or $\Phi-$ a total of over 180 megabytes of memory.

3.3 New algorithm for calculating system and survival signatures

A new efficient algorithm that outputs a multidimensional array representation of the survival signature, $\Phi$, for a system is presented in this section. For the case of a single component type, i.e. $K=1$, an array representing the system signature $q$ can be derived from the array representing the survival signature $\Phi$ by applying the simple transformation given in Eqn. 6. The inputs to the algorithm are:

- A BDD representation (standard or complement edge) of the reliability structure function of the system, where the $m$ components are ordered from 0 to $m-1$, ite nodes at level $i$ in the BDD are 
labelled with Boolean variable $x_{i}$ representing the state of component $i$ in the ordering, 1-edges from a node represent survival of the labelling component, 0-edges from a node represent failure of the labelling component, the terminal 1 node represents system survival and the terminal 0 node represents system failure.

- An associative array [31] (also known as a map or dictionary data type) that associates each of the $m$ components in the system with its component type from $\{1, \ldots, K\}$.

The algorithm computes multidimensional arrays representing $\bar{\Phi}$ for nodes at successively lower levels of the BDD, starting with the terminal nodes at level $m$ and finishing with the root node. The array representing $\bar{\Phi}$ for each ite node is computed using the results from its two child nodes, representing the positive and negative sub-parts of the Shannon decomposition of the Boolean function represented by the node (see Eqn. 9). These result are stored in an associative array and retrieved whenever the node is reencountered during the computation process. The algorithm therefore follows the dynamic programming paradigm [32] in which a problem is solved by identifying a collection of sub-problems, solving each sub-problem using the solutions to its sub-problems, iteratively solving the sub-problems in order of increasing size and storing the subproblem solutions and then retrieving them instead of resolving them when shared sub-problems are reencountered. An array representing the survival signature $\Phi$ is then computed by normalising the array representing $\bar{\Phi}$ for the complete BDD using an array representing $|S|$.

One unary operation and five binary operations and on multidimensional arrays will now be defined that are used in the algorithm. The unary operation on an array is named here as the complement operation and is only required where complement BDDs are used. The complement operation on array $A$, denoted $X^{\ddagger}$, outputs an array $B$ where the value at each index $\left(l_{1}, \ldots, l_{K}\right)$ is equal to $\prod_{k=1}^{K}\left(\begin{array}{c}L_{k}-1 \\ l_{k}\end{array}\right)$ minus the value at that index in array $A$, where $L_{k}$ is the length of array $A$ in dimension $k$. The first three binary operations are defined for pairs of multidimensional arrays with the same number of dimensions and dimension lengths. They are named here as the elementwise addition, elementwise subtraction and elementwise division operations. The elementwise addition of two arrays $A$ and $B$, denoted $A \oplus B$, outputs an array $C$ with the same number of dimensions and dimension lengths as arrays $A$ and $B$ where the value at each index in array $C$ is equal to the sum of the values at the same index in arrays $A$ and $B$. Elementwise subtraction of array $B$ from array $A$, denoted $A \ominus B$, outputs an array $C$ with the same number of dimensions and dimension lengths as arrays $A$ and $B$ where the value at index each index in array $C$ is equal to the value at that index in array $A$ minus the value at that index in array $B$. Elementwise division of array $A$ by array $\mathrm{B}$, denoted $A \oslash B$, outputs an array $C$ with the same number of dimensions and dimension lengths as arrays $A$ and $B$ where the value at index in array $C$ is equal to the value at that index in array $A$ divided by the value at that index in array $B$. The final two binary operations are defined for a first argument of a multidimensional array with $K$ dimensions and a second argument of an integer $k \in\{1, \ldots, K\}$. They are named here as the resize-k and shift-k operations. The resize-k operation on array $A$ by integer $k$ is denoted $A \boxplus k$. It returns an array $B$ with the same number of dimensions and dimension lengths as $A$, except with the length of dimension $k$ increased by 1 to length $L$, where:

- the value in $B$ at each index $\left(l_{1}, \ldots, l_{k}, \ldots, l_{K}\right)$, except where $l_{k}=L$, is equal to the value from $A$ at the same index.

- the value in $B$ at each index $\left(l_{1}, \ldots, L, \ldots, l_{K}\right)$ is equal to 0.

The shift-k operation on array $A$ by integer $k$ is denoted $A \circledast k$. It returns an array $B$ with the same number of dimensions and dimension lengths as $A$, except with the length of dimension $k$ increased by 1 , where:

- the value in $B$ at each index $\left(l_{1}, \ldots, l_{k}, \ldots, l_{K}\right)$, except where $l_{k}=0$, is equal to the value from $A$ at index $\left(l_{1}, \ldots, l_{k}-1, \ldots, l_{K}\right)$; 
- the value in $B$ at each index $\left(l_{1}, \ldots, 0, \ldots, l_{K}\right)$ is equal to 0 .

The main routine of the algorithm is shown in Figure 2. Arrays representing $\bar{\Phi}$ for the terminal nodes are computed first. A BDD comprising of only the terminal 1 node represents a Boolean function on 0 components of each type that evaluates to 1 (i.e. system survival). Therefore the array representing $\bar{\Phi}$ for the terminal 1 node has $K$ dimensions of length 1 and a single element of 1 . Conversely, a BDD comprising of only a terminal 0 node represents a Boolean function on 0 components of the $K$ components type that evaluates to 0 (i.e. system failure). Therefore the array representing $\bar{\Phi}$ for the terminal 0 node has $K$ dimensions of length 1 and a single element of 0 . For example, the arrays representing $\bar{\Phi}$ for the terminal 1 and terminal 0 nodes from the BDD in Figure 1b, which corresponds to a system with two component types (i.e. $K=2$ ) with the success tree depicted in Figure 1a, are [[1]] and [[0]] respectively. The results for $\bar{\Phi}$ corresponding to the terminal nodes are then stored in an associative array that represents a cache of all computed node results.

Arrays representing $\bar{\Phi}$ for ite nodes in the BDD at successive levels are then computed, starting with those at level $m-1$ adjacent to the terminal nodes. For an ite node labelled with Boolean variable $x_{i}$, an array representing $\bar{\Phi}$ for the positive and negative parts of the Shannon decomposition the node represents, i.e. $\left(\left(x_{i}=1\right) \wedge f_{x_{i}=1}\right)$ and $\left(\left(x_{i}=0\right) \wedge f_{x_{i}=0}\right)$ from Eqn. 9 respectively, are computed first. The subroutines of the algorithm for computing the positive and negative parts of the Shannon decomposition are shown in Figure 3 and Figure 4 respectively. To compute the array representing $\bar{\Phi}$ for the positive part, the array representing $\bar{\Phi}$ for the positive Shannon cofactor, i.e. $f_{x_{i}=1}$, is computed first. This array is computed from the array representing $\bar{\Phi}$ for the child node connected to the 1-edge of the ite node which is retrieved from the cache of previously computed results. Due to the removal of isomorphic child nodes in the BDD construction process, the path from the ite node to the child node may skip levels in the BDD where the state of the components at those levels in the ordering do not influence the value of $f_{x_{i}=1}$. The state vector counts in the array from the child node are therefore updated by accounting for the survival and failure of the component corresponding to each missing level. The operation $\bar{\Phi} \boxplus \mathrm{k}$ is used to update the state vector counts in $\bar{\Phi}$ to account for an additional component of type $k$ that fails whilst the operation $\bar{\Phi} \circledast \mathrm{k}$ is used to update the state vector counts in $\bar{\Phi}$ to account for an additional component of type $k$ that survives. The elementwise addition of the resultant arrays from these two operations therefore gives an array representing $\bar{\Phi}$ that is updated for a component of type $k$ labelling a level skipped by an edge. The array representing $\bar{\Phi}$ for the negative part is computed in a similar way, except that array computed for the node connected to the 0-edge of the ite node rather than the 1-edge is retrieved from the cache, the complement operation $\neq$ is applied to the array if the 0 -edge is complemented, and the $\boxplus$ operation is used instead of $\circledast$ in the final step to update the state vector counts for the failure rather than survival of the component labelling the ite node. Elementwise addition of the arrays representing $\bar{\Phi}$ for the positive and negative parts is then performed to obtain an array representing $\bar{\Phi}$ with the total state vector counts for the ite node. The result for $\bar{\Phi}$ corresponding to the ite node is then stored in the associative array that represents a cache of all computed node results. 


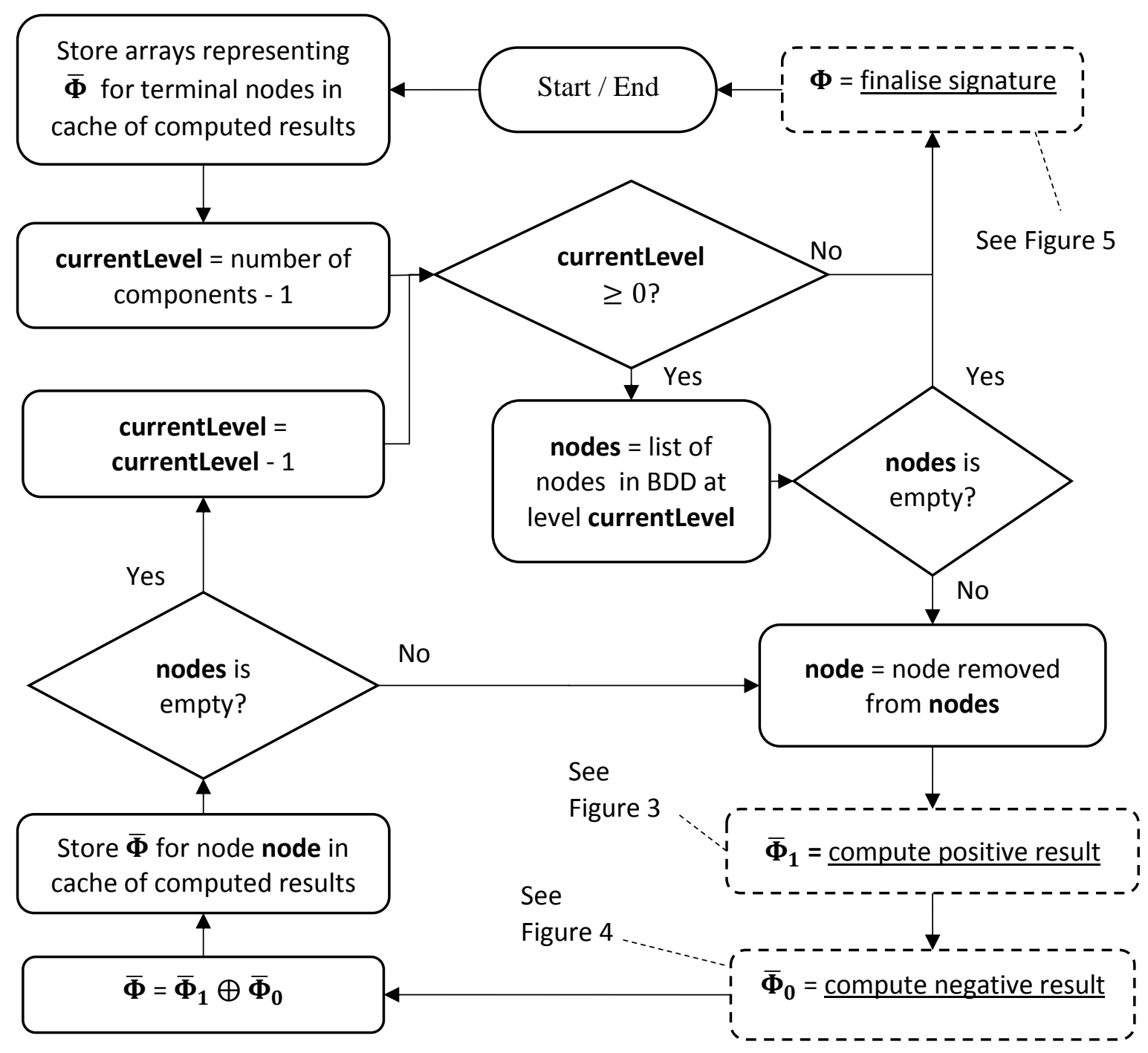

Figure 2 - Main routine of the algorithm that computes survival signatures.

Once the array representing $\bar{\Phi}$ for the root node in the BDD has been computed, the subroutine shown in Figure 5 is used to update the array to account for remaining levels in the BDD, i.e. where the root node is at a level greater than 0 , and obtain the survival signature through application of a normalisation step. Due to the removal of isomorphic child nodes in the BDD construction process, a root node at a level greater than 0 signifies that the survival or failure of the components at lower levels in the ordering do not influence whether or not the system survives. Updating the array representing $\bar{\Phi}$ to account for such levels follows the same process as for levels skipped in the edge path between an ite node and its child node. For the purpose of normalisation, an array representing $|\mathrm{S}|$ is created with $K$ dimensions and length $M_{k}+1$ in dimension $k$, with the value at each index $\left(l_{1}, \ldots, l_{k}, \ldots, l_{K}\right)$ set equal to $\prod_{k=1}^{K}\left(\begin{array}{c}M_{k} \\ l_{k}\end{array}\right)$. Elementwise division of the array representing $\bar{\Phi}$ by the array representing $|S|$ is then performed to derive the array representing the survival signature $\Phi$, which is the final result output by the algorithm. 


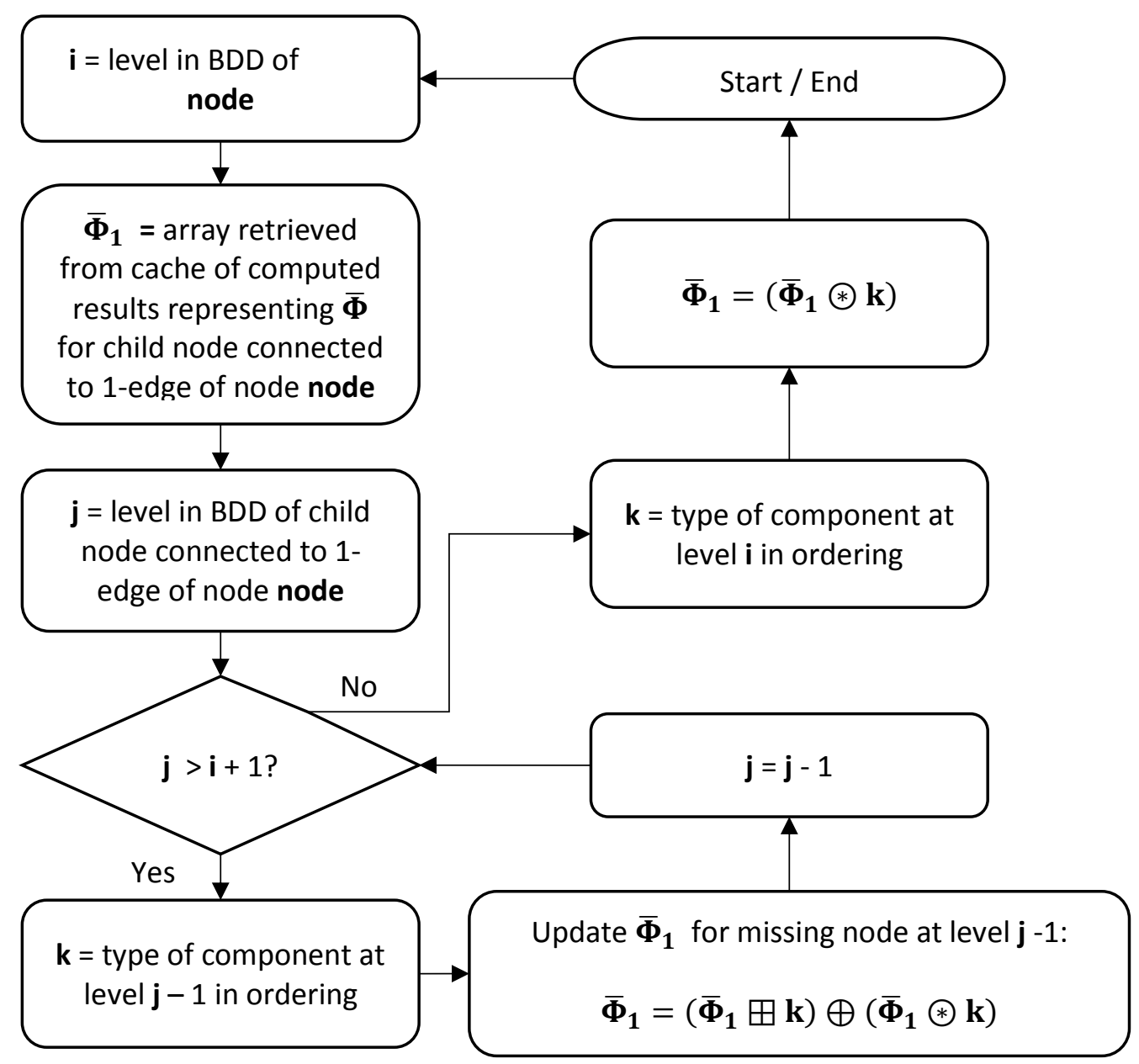

Figure 3 - "compute positive result" subroutine of the algorithm that computes $\bar{\Phi}$ for positive part of Shannon decomposition of an ite node. 


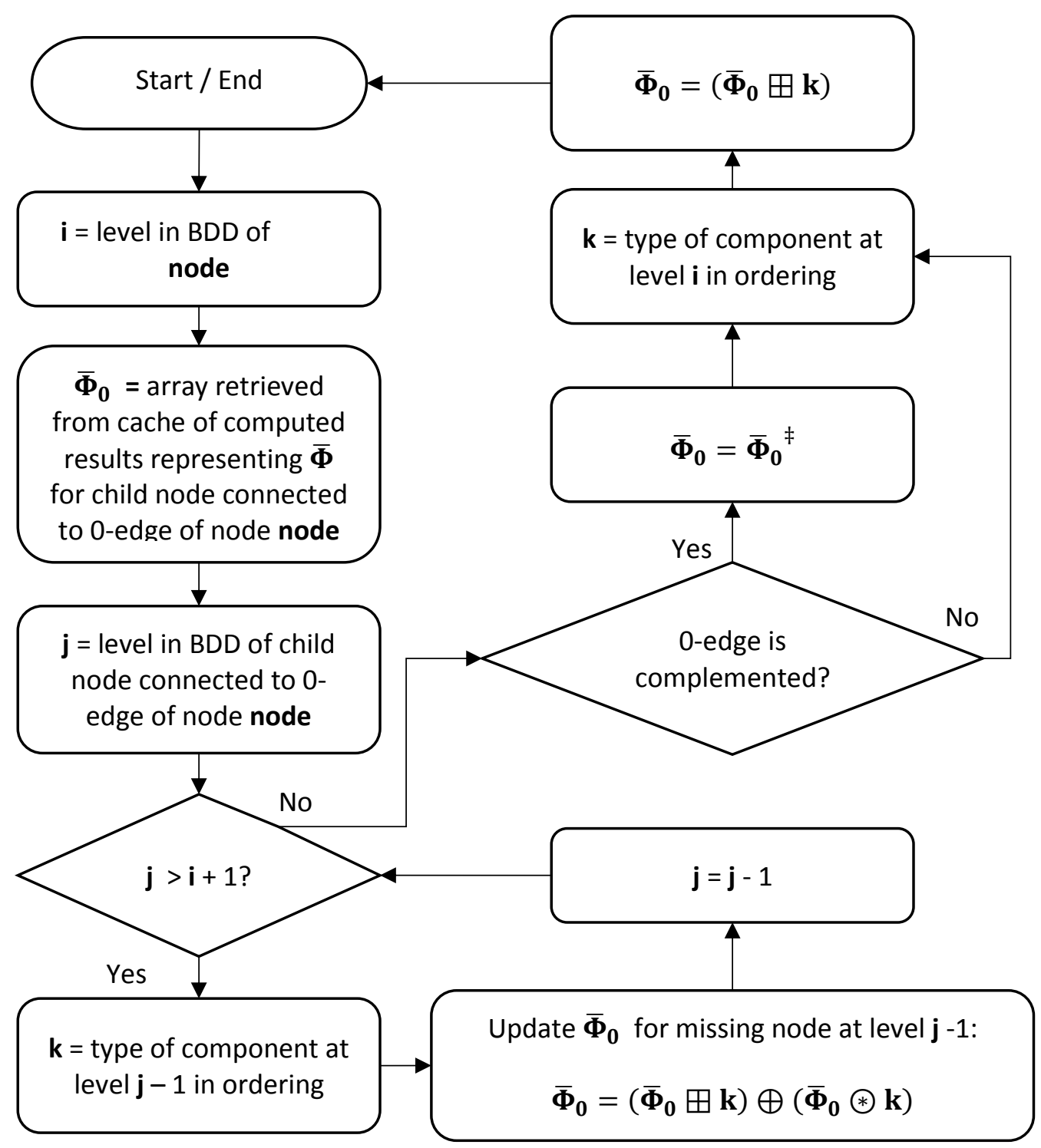

Figure 4 - "compute negative result" subroutine of the algorithm that computes $\bar{\Phi}$ for negative part of Shannon decomposition of an ite node. 


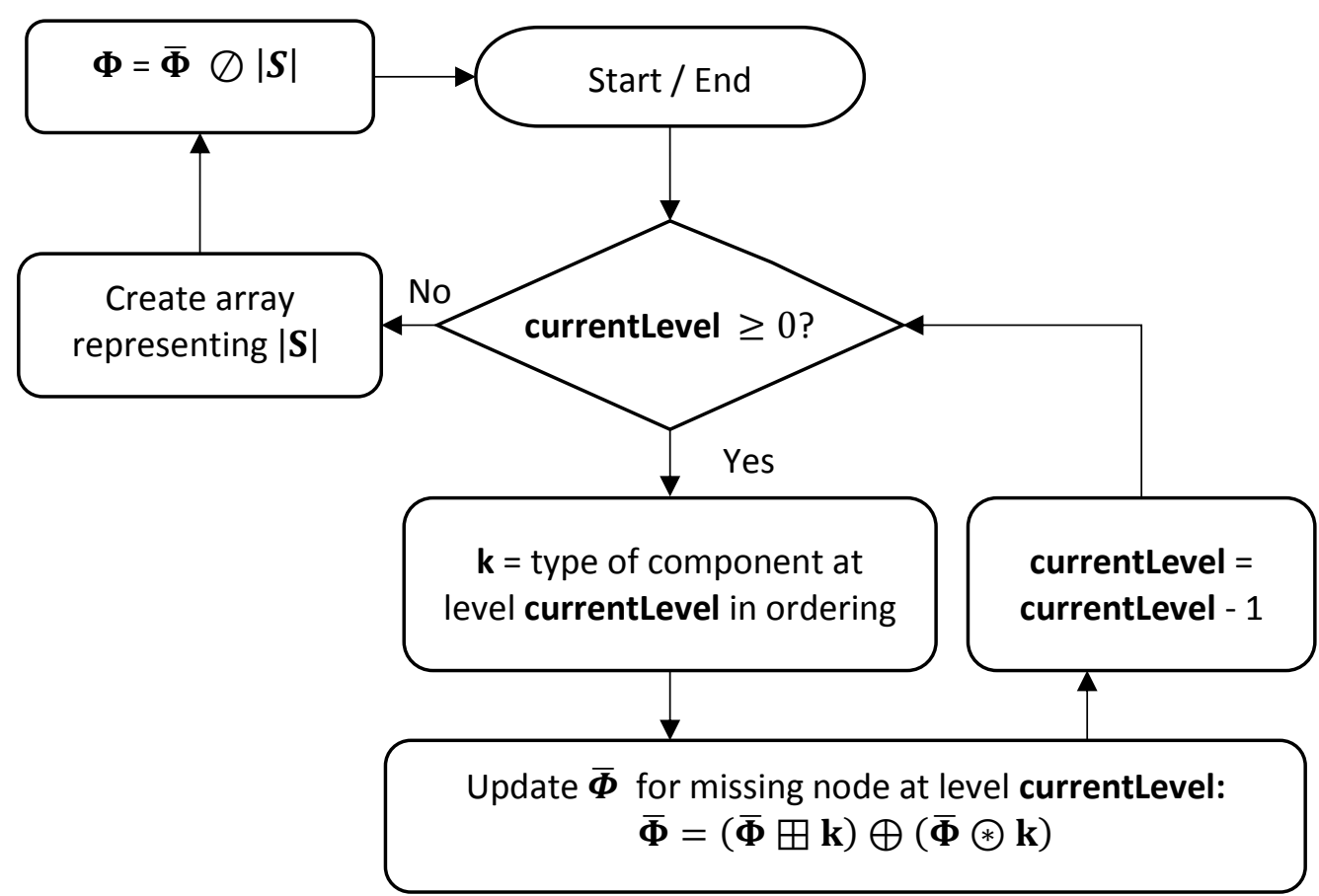

Figure 5 - "finalise signature" subroutine of the algorithm that finalises the computation of a survival signature.
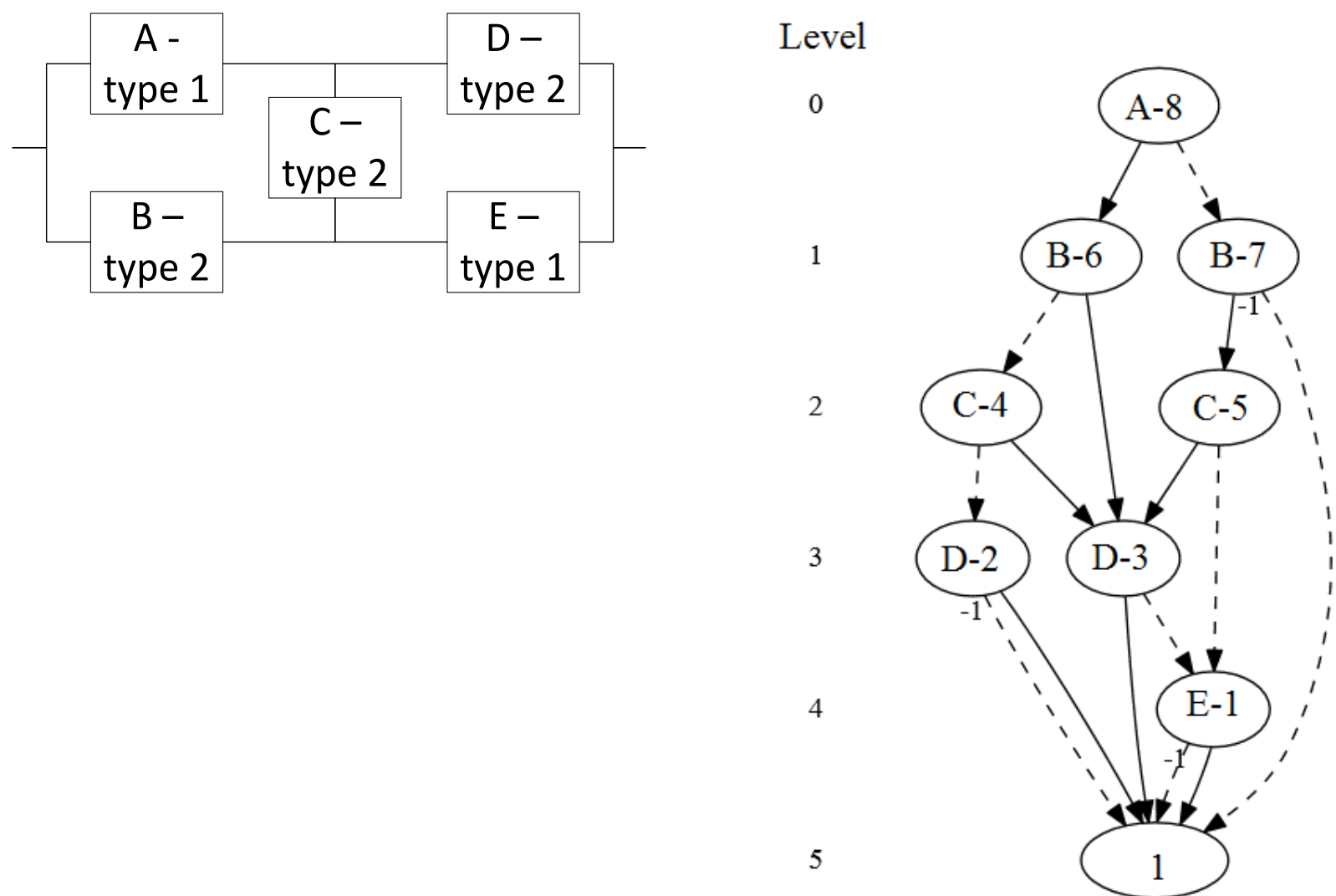

Figure 6a - Reliability block diagram for an example five component system with two component types, where each node is labelled with the component and component type.
Figure 6b - BDD for the example system shown in Figure 6a, where each ite node is labelled with the component and node number. 


\subsection{Example Computation}

Consider a five component system (consisting of components A, B, C, D and E) with two component types (consisting of types 1 and 2) and the reliability structure given by the reliability block diagram shown in Figure $6 \mathrm{a}$, where each node is labelled with the component and component type. The corresponding BDD constructed with components ordered alphabetically is shown in Figure $6 \mathrm{~b}$, where each ite node is labelled with the component and node number.

The multidimensional arrays representing $\bar{\Phi}$ for the terminal 1 and terminal 0 nodes of this system are [[1]] and $[[0]]$ respectively. The multidimensional arrays representing $\bar{\Phi}$ for each of the ite nodes in the BDD are given in Table 1.

Table 1 - Multidimensional arrays representing $\bar{\Phi}$ for each ite node from the BDD shown in Figure $6 \mathrm{~b}$.

\begin{tabular}{c|c|c|c}
$\begin{array}{c}\text { ite node } \\
\text { from } \\
\text { BDD }\end{array}$ & $\begin{array}{c}\text { Multidimensional array } \\
\text { representing } \overline{\mathbf{\Phi}} \text { for positive part } \\
\text { of Shannon decomposition }\end{array}$ & $\begin{array}{c}\text { Multidimensional array } \\
\text { representing } \overline{\mathbf{\Phi}} \text { for negative part } \\
\text { of Shannon decomposition }\end{array}$ & $\begin{array}{c}\text { Multidimensional array } \\
\text { representing } \overline{\mathbf{\Phi}}\end{array}$ \\
\hline 1 & {$[[0],[1]]$} & {$[[0],[0]]$} & {$[[0],[1]]$} \\
2 & {$[[0,1],[0,1]]$} & {$[[0,0],[0,0]]$} & {$[[0,1],[0,1]]$} \\
3 & {$[[0,0],[1,0]]$} & {$[[0,1],[0,1]]$} & {$[[0,1],[1,1]]$} \\
4 & {$[[0,0,1],[0,1,1]]$} & {$[[0,1,0],[0,1,0]]$} & {$[[0,1,1],[0,2,1]]$} \\
5 & {$[[0,0,1],[0,1,1]]$} & {$[[0,0,0],[1,1,0]]$} & {$[[0,0,1],[1,2,1]]$} \\
6 & {$[[0,0,1,1],[0,1,2,1]]$} & {$[[0,1,1,0],[0,2,1,0]]$} & {$[[0,1,2,1],[0,3,3,1]]$} \\
7 & {$[[0,0,0,1],[0,1,2,1]]$} & {$[[0,0,0,0],[0,0,0,0]]$} & {$[[0,0,0,1],[0,1,2,1]]$} \\
8 & {$[[0,0,0,0],[0,1,2,1],[0,3,3,1]]$} & {$[[0,0,0,1],[0,1,2,1],[0,0,0,0]]$} & {$[[0,0,0,1],[0,2,4,2],[0,3,3,1]]$}
\end{tabular}

The array representing $|S|$ is:

$|S|=[[1,3,3,1],[2,6,6,2],[1,3,3,1]]$

Finally, the multidimensional array representation of the survival signature $\Phi$ for the BDD obtained through elementwise division of the array representing $\bar{\Phi}$ for the $\mathrm{BDD}$ (i.e. the result for the root node of the BDD, ite node 8 , given from Table 1 ) by the array representing $|S|$ is:

$\Phi=[[0,0,0,1],[0,2,4,2],[0,3,3,1]] \oslash[[1,3,3,1],[2,6,6,2],[1,3,3,1]]=\left[[0,0,0,1],\left[0, \frac{1}{3}, \frac{2}{3}, 1\right],[0,1,1,1]\right]$

4 Application of New Method for System and Survival Signature to Benchmark Problems The algorithm presented in this paper has been implemented as a computer code (available on request) in the Python programming language, using the NumPy open source library [33] to provide an efficient implementation of multi-dimensional arrays and array operations. The algorithm and its implementation were validated for correctness by verifying computed signatures for a set of small network reliability problems (which contained 22 components or less) against results obtained from the ReliabilityTheory package [19] for the $\mathrm{R}$ programming language [20]. Additional verification was performed by using Eqn. 5 to calculate the probabilities of system survival from the computed survival signatures and comparing them against the known values for each system.

To demonstrate the efficiency of the algorithm, it was used to compute the signatures from the fault tree representation of the reliability structure for 7 systems of different size and complexity. Systems 1, 2 and 3 
were composed by the author, whilst the European 1 and European 3 systems were obtained from the literature [34] and have been used previously by other researchers as benchmarks [24]. European 1 and European 3 systems were chosen since they are large and complex systems derived from practice that feature many cutsets (46188 and 24386 respectively) and components (61 and 80 respectively). Finally, two further systems named European 1a and European 1b were derived from the European 1 system by reducing the number of component types (from the original 14 types to 3 and 6 respectively). The fault tree and component types for system 1 are given in Table A.1 and Table A.2, respectively, of Appendix A. A BDD for each of the seven fault trees was computed using the method from Rauzy [24] with components ordered according to the sequence in which they were first encountered during a depth first traversal [35] of the fault tree starting at the top event.

Table 3 presents the results for computing signatures for each of the example systems, using both the new algorithm that was presented in Section 3 and an enumerative algorithm similar to that described in Section 2. The results were obtained using a computer with an Intel i3-4130 3.4GHz CPU and 8GB RAM. In Appendix $\mathrm{B}$, the survival signature that was computed for system 1 is shown in Table B.1a and Table B.1b, whilst the system signature for the European 3 system is shown in Table B.2.

Table 3 - Results for computing signatures using new algorithm and enumerative algorithm for example systems (all times given in seconds)

\begin{tabular}{|c|c|c|c|c|c|c|c|}
\hline System & 1 & 2 & 3 & $\begin{array}{c}\text { European } \\
\text { 1a }\end{array}$ & European 1b & European 1 & European 3 \\
\hline Number Components & 16 & 21 & 23 & 61 & 61 & 61 & 80 \\
\hline $\begin{array}{l}\text { Number } \\
\text { Component Types }\end{array}$ & 3 & 4 & 8 & 3 & 6 & 14 & 1 \\
\hline $\begin{array}{l}\text { Number of survival } \\
\text { signature elements }\end{array}$ & 180 & 1400 & 30240 & 8370 & 419238 & 745875000 & 81 \\
\hline Number BDD Nodes & 17 & 22 & 41 & 7365 & 7365 & 7365 & 11789 \\
\hline $\begin{array}{l}\text { BDD Computation } \\
\text { Time }\end{array}$ & 0.00 & 0.00 & 0.00 & 0.14 & 0.14 & 0.14 & 0.38 \\
\hline $\begin{array}{l}\text { Signature } \\
\text { Computation Time } \\
\text { (New Algorithm) }\end{array}$ & 0.00 & 0.01 & 0.01 & 0.93 & 3.53 & $\mathrm{n} / \mathrm{a}$ & 1.02 \\
\hline $\begin{array}{l}\text { Signature } \\
\text { Computation } \\
\text { (Enumerative } \\
\text { Algorithm) }\end{array}$ & 0.14 & 27.42 & 127.56 & $\mathrm{n} / \mathrm{a}$ & $\mathrm{n} / \mathrm{a}$ & $\mathrm{n} / \mathrm{a}$ & $\mathrm{n} / \mathrm{a}$ \\
\hline
\end{tabular}

The results given in Table 3 demonstrate that the new algorithm is extremely efficient, computing signatures for all the systems in less than 4 seconds, with the exception of the full European 1 system that has 14 component types. In contrast, the enumerative algorithm used for comparison purposes could not obtain results for any of the European systems in reasonable times and took over 2 minutes to compute the survival signature for System 3 which took only 0.01 seconds with the new algorithm. The results also show that the new algorithm scales well with the complexity of the analysed system, for example it was able to compute the survival signature for systems 2 and 3 in the same time despite System 3 having an extra 2 components and twice as many component types. In comparison, the enumerative algorithm took more than 4 times longer to compute the survival signature for System 3 than it did for System 2. Even for very complex systems the new algorithm remains efficient, for example it took just over 1 second to compute the system signature for the European 3 system with 80 components. The only system for which it was not possible to compute a signature 
was for the full European fault tree 1 with all 14 component types. For this system, the array representation of the survival signature has over 0.75 billion elements and the memory required to represent it in RAM exceeded the resources available on the computer used to perform the analysis.

\section{$5 \quad$ Summary and Conclusions}

The system signature and survival signature are valuable tools for the analysis and assessment of system reliability as evidenced by the wide range of theoretical applications found in the literature. These applications include comparing the reliability of different system designs and determining where redundancy should be added to a system to maximise gains in reliability. It is well known that the computation of signatures is very challenging and the previously published approaches are only feasible for simple systems with few components. The development of algorithms that are able to compute signatures for complex systems with large numbers of components is therefore important so that they can be analysed with the many signature based reliability analysis methods that have been published in the literature.

A new algorithm for computing system and survival signatures was introduced, based on the use of binary decision diagrams (BDDs), multidimensional arrays and the dynamic programming paradigm. Results were presented comparing the efficiency of the new algorithm with an enumerative algorithm in the computation of signatures for a range of benchmark problems that are representative of complex real world systems. These demonstrated that the new algorithm results in significantly increased efficiency and scalability with increasing system complexity. For example, the survival signature for an example system with 23 components and 8 component types was computed in just 0.01 seconds by the new algorithm whereas the enumerative algorithm took over 2 minutes. The new algorithm also computed signatures for 3 out of 4 of benchmark systems from the literature (ranging from 61 and 80 components), whilst the enumerative algorithm was unable to compute signatures for any of them in reasonable times. The presented approach will therefore permit system and survival signatures to be computed for many large and complex systems for which this was infeasible with previous approaches. This should result in greater practical application of the theoretical uses for signatures in analysing the reliability that have been developed, such as the methods for stochastic comparison of the reliability of alternative system designs.

A limitation of the algorithm is that it relies on the BDD representation of the reliability structure of the system to be analysed. The ability to compute this data structure depends on the representation of the system reliability structure available for the system to be analysed. For example, they can easily be computed for even very large fault trees since they represent the reliability structure directly in terms of the Boolean logic, however it is more difficult for very large networks where the reliability structure must be computed. Furthermore, the memory requirements for the computational representation of signatures was discussed in the paper and showed that huge amounts of memory can be required for certain systems with many components and component types. For this reason, the implementation of the algorithm was unable to compute the survival signature for one of the example benchmark systems as the RAM required to represent its signature exceeded the available resources on the computer used for the analysis. Therefore implementations of the algorithm that are able to manipulate the arrays representing signatures without requiring complete storage in RAM are necessary to analyse such cases. The development of algorithms and implementations to overcome these limitations are areas for future research.

[1] Samaniego FJ. On Closure of the IFR Class Under Formation of Coherent Systems. IEEE Trans Reliab 1985;R-34:69-72. doi:10.1109/TR.1985.5221935.

[2] Samaniego FJ. System Signatures and their Applications in Engineering Reliability. vol. 110. Boston, MA: Springer US; 2007. doi:10.1007/978-0-387-71797-5. 
[3] Boland PJ, Samaniego FJ. The Signature of a Coherent System and Its Applications in Reliability. In: Soyer R, Mazzuchi TA, Singpurwalla ND, editors. Math. Reliab. An Expo. Perspect., Boston, MA: Springer US; 2004, p. 3-30. doi:10.1007/978-1-4419-9021-1_1.

[4] Block H, Dugas MR, Samaniego FJ. Characterizations of the Relative Behavior of Two Systems via Properties of Their Signature Vectors. In: Balakrishnan N, Sarabia JM, Castillo E, editors. Adv. Distrib. Theory, Order Stat. Inference, Boston, MA: Birkh\{ä\}user Boston; 2006, p. 279-89. doi:10.1007/0-81764487-3_18.

[5] Eryilmaz S. Review of recent advances in reliability of consecutive $k$-out-of- $n$ and related systems. Proc Inst Mech Eng Part O J Risk Reliab 2010;224:225-37. doi:10.1243/1748006XJRR332.

[6] Coolen FPA, Coolen-Maturi T. Generalizing the Signature to Systems with Multiple Types of Components. Complex Syst. Dependability, Springer Berlin Heidelberg; 2012, p. 115-30. doi:10.1007/9783-642-30662-4_8.

[7] Pa Coolen F, Coolen-Maturi T, Al-Nefaiee AH. Nonparametric predictive inference for system reliability using the survival signature. Proc IMechE Part O J Risk Reliab 2014;228:437-48. doi:10.1177/1748006X14526390.

[8] Aslett LJM, Coolen FPA, Wilson SP. Bayesian Inference for Reliability of Systems and Networks Using the Survival Signature. Risk Anal 2015;35:1640-51. doi:10.1111/risa.12228.

[9] Coolen FPA, Coolen-Maturi T. Modelling Uncertain Aspects of System Dependability with Survival Signatures. Dependability Probl. Complex Inf. Syst., Springer International Publishing; 2015, p. 19-34. doi:10.1007/978-3-319-08964-5_2.

[10] Coolen FPA, Coolen-Maturi T. Predictive inference for system reliability after common-cause component failures. Reliab Eng Syst Saf 2015;135:27-33. doi:10.1016/j.ress.2014.11.005.

[11] Feng G, Patelli E, Beer M, Coolen FPA. Imprecise system reliability and component importance based on survival signature. Reliab Eng Syst Saf 2016;150:116-25. doi:10.1016/j.ress.2016.01.019.

[12] Walter G, Aslett LJM, Coolen FPA. Bayesian Nonparametric System Reliability using Sets of Priors. arXiv:160201650 [statME] 2016.

[13] Samaniego F, Navarro J. On comparing coherent systems with heterogeneous components. Adv Appl Probab 2016;48:88-111.

[14] Kochar S, Mukerjee H, Samaniego FJ. The "signature" of a coherent system and its application to comparisons among systems. Nav Res Logist 1999;46:507-23. doi:10.1002/(SICI)15206750(199908)46:5<507::AID-NAV4>3.0.CO;2-D.

[15] Boland PJ. Signatures of Indirect Majority Systems. J Appl Probab 2001;38:597-603.

[16] Marichal J-L, Mathonet P. Computing system signatures through reliability functions. Stat Probab Lett 2013;83:710-7. doi:http://dx.doi.org/10.1016/j.spl.2012.11.018.

[17] Triantafyllou IS, Koutras M V. On the signature of coherent systems and applications. Probab Eng Informational Sci 2008;22:19-35. doi:10.1017/S0269964808000028.

[18] Da G, Zheng B, Hu T. On computing signatures of coherent systems. J Multivar Anal 2012;103:14250. doi:http://dx.doi.org/10.1016/j.jmva.2011.06.015.

[19] Aslett LJM. ReliabilityTheory: Tools for structural reliability analysis, R package 2012.

[20] R Core Team. R: A Language and Environment for Statistical Computing 2015. 
[21] Bryant RE. Graph-Based Algorithms for Boolean Function Manipulation. IEEE Trans Comput 1986;C-35:677-91. doi:10.1109/TC.1986.1676819.

[22] Shannon CE. A symbolic analysis of relay and switching circuits. Trans Am Inst Electr Eng 1938;57:713-23. doi:10.1109/T-AIEE.1938.5057767.

[23] Brace KS, Rudell RL, Bryant RE. Efficient Implementation of a BDD Package. Proc. 27th ACM/IEEE Des. Autom. Conf., New York, NY, USA: ACM; 1990, p. 40-5. doi:10.1145/123186.123222.

[24] Rauzy A. New algorithms for fault trees analysis. Reliab Eng Syst Saf 1993;40:203-11.

[25] Andrews JD, Dunnett SJ. Event-tree analysis using binary decision diagrams. IEEE Trans Reliab 2000;49:230-8. doi:10.1109/24.877343.

[26] Hardy G, Lucet C, Limnios N. K-Terminal Network Reliability Measures With Binary Decision Diagrams. IEEE Trans Reliab 2007;56:506-15. doi:10.1109/TR.2007.898572.

[27] Bjorkman K. Solving dynamic flowgraph methodology models using binary decision diagrams. Reliab Eng Syst Saf 2013;111:206-16. doi:10.1016/j.ress.2012.11.009.

[28] Bollig B, Wegener I. Improving the variable ordering of OBDDs is NP-complete. IEEE Trans Comput 1996;45:993-1002. doi:10.1109/12.537122.

[29] Huang H-Z, Zhang H, Li Y. A New Ordering Method of Basic Events in Fault Tree Analysis. Qual Reliab Eng Int 2012;28:297-305. doi:10.1002/qre.1245.

[30] Manoj S, Dr. GS, Dr. RKC. A New Approach for Finding the various Optimal Variable Ordering to Generate the Binary Decision Diagrams (BDD) of a Computer Communication Network. Int J Comput Appl 2011;31:1-8.

[31] Mehlhorn K, Sanders P. Algorithms and Data Structures: The Basic Toolbox. Springer-Verlag Berlin Heidelberg; 2008. doi:10.1007/978-3-540-77978-0.

[32] Skiena SS. Dynamic Programming. Algorithm Des. Man., London: Springer London; 2012, p. $273-$ 315. doi:10.1007/978-1-84800-070-4_8.

[33] van der Walt S, Colbert SC, Varoquaux G. The NumPy array: a structure for efficient numerical computation. CoRR 2011;abs/1102.1.

[34] Limnios N. Fault Trees. Fault Trees, Wiley-ISTE; 2010.

[35] Cormen TH, Leiserson CE, Rivest R, Stein C. Introduction to Algorithms. 3rd ed. MIT Press; 2009. 
Appendix A - Description of system 1.

Table A.1 - Fault tree description for system 1.

\begin{tabular}{l|l|l} 
Gate Name & Logical Operator & Inputs \\
\hline G1 & AND & G2, G3, G4 \\
G2 & OR & G5, BE1, BE2 \\
G3 & OR & G6, BE3 \\
G4 & OR & G7, G8 \\
G5 & AND & G9, G10 \\
G6 & AND & BE4, BE5 \\
G7 & AND & BE6, BE7, BE8 \\
G8 & AND & BE9, BE10 \\
G9 & OR & BE11, BE12, BE13 \\
G10 & OR & BE14, BE15, BE16
\end{tabular}

Table A.2 - Components and component types for system 1.

\begin{tabular}{l|l} 
Component & Component Type \\
\hline BE1 & 1 \\
BE2 & 2 \\
BE3 & 1 \\
BE4 & 1 \\
BE5 & 3 \\
BE6 & 2 \\
BE7 & 2 \\
BE8 & 1 \\
BE9 & 1 \\
BE10 & 3 \\
BE11 & 1 \\
BE12 & 2 \\
BE13 & 2 \\
BE14 & 1 \\
BE15 & 1 \\
BE16 & 1
\end{tabular}


Appendix B - Signatures for system 1 and the European 3 system.

Table B.1a - Part 1 of survival signature for system 1.

\begin{tabular}{|c|c|c|c|c|c|c|c|}
\hline$l_{1}$ & $l_{2}$ & $l_{3}$ & $\begin{array}{l}\text { Survival } \\
\text { signature } \\
\text { value } \Phi_{l_{1}, l_{2}, l_{3}}\end{array}$ & $l_{1}$ & $l_{2}$ & $l_{3}$ & $\begin{array}{l}\text { Survival } \\
\text { signature } \\
\text { value } \\
\Phi_{l_{1}, l_{2}, l_{3}}\end{array}$ \\
\hline 9 & 5 & 2 & 1.0000 & 7 & 2 & 2 & 0.9972 \\
\hline 9 & 5 & 1 & 1.0000 & 7 & 2 & 1 & 0.9875 \\
\hline 9 & 5 & 0 & 1.0000 & 7 & 2 & 0 & 0.9611 \\
\hline 9 & 4 & 2 & 1.0000 & 7 & 1 & 2 & 0.9889 \\
\hline 9 & 4 & 1 & 1.0000 & 7 & 1 & 1 & 0.9722 \\
\hline 9 & 4 & 0 & 1.0000 & 7 & 1 & 0 & 0.9333 \\
\hline 9 & 3 & 2 & 1.0000 & 7 & 0 & 2 & 0.9722 \\
\hline 9 & 3 & 1 & 1.0000 & 7 & 0 & 1 & 0.9444 \\
\hline 9 & 3 & 0 & 1.0000 & 7 & 0 & 0 & 0.8889 \\
\hline 9 & 2 & 2 & 1.0000 & 6 & 5 & 2 & 1.0000 \\
\hline 9 & 2 & 1 & 1.0000 & 6 & 5 & 1 & 0.9940 \\
\hline 9 & 2 & 0 & 1.0000 & 6 & 5 & 0 & 0.9762 \\
\hline 9 & 1 & 2 & 1.0000 & 6 & 4 & 2 & 1.0000 \\
\hline 9 & 1 & 1 & 1.0000 & 6 & 4 & 1 & 0.9798 \\
\hline 9 & 1 & 0 & 1.0000 & 6 & 4 & 0 & 0.9214 \\
\hline 9 & 0 & 2 & 1.0000 & 6 & 3 & 2 & 0.9988 \\
\hline 9 & 0 & 1 & 1.0000 & 6 & 3 & 1 & 0.9690 \\
\hline 9 & 0 & 0 & 1.0000 & 6 & 3 & 0 & 0.8857 \\
\hline 8 & 5 & 2 & 1.0000 & 6 & 2 & 2 & 0.9821 \\
\hline 8 & 5 & 1 & 1.0000 & 6 & 2 & 1 & 0.9399 \\
\hline 8 & 5 & 0 & 1.0000 & 6 & 2 & 0 & 0.8369 \\
\hline 8 & 4 & 2 & 1.0000 & 6 & 1 & 2 & 0.9571 \\
\hline 8 & 4 & 1 & 1.0000 & 6 & 1 & 1 & 0.9024 \\
\hline 8 & 4 & 0 & 1.0000 & 6 & 1 & 0 & 0.7857 \\
\hline 8 & 3 & 2 & 1.0000 & 6 & 0 & 2 & 0.9167 \\
\hline 8 & 3 & 1 & 1.0000 & 6 & 0 & 1 & 0.8452 \\
\hline 8 & 3 & 0 & 1.0000 & 6 & 0 & 0 & 0.7143 \\
\hline 8 & 2 & 2 & 1.0000 & 5 & 5 & 2 & 1.0000 \\
\hline 8 & 2 & 1 & 1.0000 & 5 & 5 & 1 & 0.9643 \\
\hline 8 & 2 & 0 & 1.0000 & 5 & 5 & 0 & 0.8651 \\
\hline 8 & 1 & 2 & 1.0000 & 5 & 4 & 2 & 1.0000 \\
\hline 8 & 1 & 1 & 1.0000 & 5 & 4 & 1 & 0.9405 \\
\hline 8 & 1 & 0 & 1.0000 & 5 & 4 & 0 & 0.7873 \\
\hline 8 & 0 & 2 & 1.0000 & 5 & 3 & 2 & 0.9929 \\
\hline 8 & 0 & 1 & 1.0000 & 5 & 3 & 1 & 0.9175 \\
\hline 8 & 0 & 0 & 1.0000 & 5 & 3 & 0 & 0.7333 \\
\hline 7 & 5 & 2 & 1.0000 & 5 & 2 & 2 & 0.9548 \\
\hline 7 & 5 & 1 & 1.0000 & 5 & 2 & 1 & 0.8647 \\
\hline 7 & 5 & 0 & 1.0000 & 5 & 2 & 0 & 0.6690 \\
\hline 7 & 4 & 2 & 1.0000 & 5 & 1 & 2 & 0.9048 \\
\hline 7 & 4 & 1 & 0.9972 & 5 & 1 & 1 & 0.8016 \\
\hline 7 & 4 & 0 & 0.9889 & 5 & 1 & 0 & 0.6032 \\
\hline 7 & 3 & 2 & 1.0000 & 5 & 0 & 2 & 0.8333 \\
\hline 7 & 3 & 1 & 0.9944 & 5 & 0 & 1 & 0.7143 \\
\hline 7 & 3 & 0 & 0.9778 & 5 & 0 & 0 & 0.5159 \\
\hline
\end{tabular}


Table B.1b - Part 2 of survival signature for system 1.

\begin{tabular}{|c|c|c|c|c|c|c|c|}
\hline$l_{1}$ & $l_{2}$ & $l_{3}$ & $\begin{array}{l}\text { Survival } \\
\text { signature value } \\
\left(\Phi_{l_{1}, l_{2}, l_{3}}\right)\end{array}$ & $l_{1}$ & $l_{2}$ & $l_{3}$ & $\begin{array}{l}\text { Survival } \\
\text { signature value } \\
\left(\Phi_{l_{1}, l_{2}, l_{3}}\right)\end{array}$ \\
\hline 4 & 5 & 2 & 1.0000 & 2 & 2 & 2 & 0.8250 \\
\hline 4 & 5 & 1 & 0.9048 & 2 & 2 & 1 & 0.5708 \\
\hline 4 & 5 & 0 & 0.6825 & 2 & 2 & 0 & 0.1917 \\
\hline 4 & 4 & 2 & 1.0000 & 2 & 1 & 2 & 0.6500 \\
\hline 4 & 4 & 1 & 0.8802 & 2 & 1 & 1 & 0.4333 \\
\hline 4 & 4 & 0 & 0.6143 & 2 & 1 & 0 & 0.1333 \\
\hline 4 & 3 & 2 & 0.9810 & 2 & 0 & 2 & 0.4167 \\
\hline 4 & 3 & 1 & 0.8452 & 2 & 0 & 1 & 0.2500 \\
\hline 4 & 3 & 0 & 0.5595 & 2 & 0 & 0 & 0.0556 \\
\hline 4 & 2 & 2 & 0.9183 & 1 & 5 & 2 & 1.0000 \\
\hline 4 & 2 & 1 & 0.7742 & 1 & 5 & 1 & 0.6111 \\
\hline 4 & 2 & 0 & 0.4952 & 1 & 5 & 0 & 0.1111 \\
\hline 4 & 1 & 2 & 0.8349 & 1 & 4 & 2 & 1.0000 \\
\hline 4 & 1 & 1 & 0.6841 & 1 & 4 & 1 & 0.6111 \\
\hline 4 & 1 & 0 & 0.4222 & 1 & 4 & 0 & 0.1111 \\
\hline 4 & 0 & 2 & 0.7222 & 1 & 3 & 2 & 0.9222 \\
\hline 4 & 0 & 1 & 0.5635 & 1 & 3 & 1 & 0.5611 \\
\hline 4 & 0 & 0 & 0.3254 & 1 & 3 & 0 & 0.1000 \\
\hline 3 & 5 & 2 & 1.0000 & 1 & 2 & 2 & 0.7667 \\
\hline 3 & 5 & 1 & 0.8214 & 1 & 2 & 1 & 0.4611 \\
\hline 3 & 5 & 0 & 0.4762 & 1 & 2 & 0 & 0.0778 \\
\hline 3 & 4 & 2 & 1.0000 & 1 & 1 & 2 & 0.5333 \\
\hline 3 & 4 & 1 & 0.8036 & 1 & 1 & 1 & 0.3111 \\
\hline 3 & 4 & 0 & 0.4333 & 1 & 1 & 0 & 0.0444 \\
\hline 3 & 3 & 2 & 0.9643 & 1 & 0 & 2 & 0.2222 \\
\hline 3 & 3 & 1 & 0.7601 & 1 & 0 & 1 & 0.1111 \\
\hline 3 & 3 & 0 & 0.3893 & 1 & 0 & 0 & 0.0000 \\
\hline 3 & 2 & 2 & 0.8750 & 0 & 5 & 2 & 1.0000 \\
\hline 3 & 2 & 1 & 0.6756 & 0 & 5 & 1 & 0.5000 \\
\hline 3 & 2 & 0 & 0.3333 & 0 & 5 & 0 & 0.0000 \\
\hline 3 & 1 & 2 & 0.7500 & 0 & 4 & 2 & 1.0000 \\
\hline 3 & 1 & 1 & 0.5595 & 0 & 4 & 1 & 0.5000 \\
\hline 3 & 1 & 0 & 0.2619 & 0 & 4 & 0 & 0.0000 \\
\hline 3 & 0 & 2 & 0.5833 & 0 & 3 & 2 & 0.9000 \\
\hline 3 & 0 & 1 & 0.4048 & 0 & 3 & 1 & 0.4500 \\
\hline 3 & 0 & 0 & 0.1667 & 0 & 3 & 0 & 0.0000 \\
\hline 2 & 5 & 2 & 1.0000 & 0 & 2 & 2 & 0.7000 \\
\hline 2 & 5 & 1 & 0.7222 & 0 & 2 & 1 & 0.3500 \\
\hline 2 & 5 & 0 & 0.2778 & 0 & 2 & 0 & 0.0000 \\
\hline 2 & 4 & 2 & 1.0000 & 0 & 1 & 2 & 0.4000 \\
\hline 2 & 4 & 1 & 0.7139 & 0 & 1 & 1 & 0.2000 \\
\hline 2 & 4 & 0 & 0.2611 & 0 & 1 & 0 & 0.0000 \\
\hline 2 & 3 & 2 & 0.9444 & 0 & 0 & 2 & 0.0000 \\
\hline 2 & 3 & 1 & 0.6653 & 0 & 0 & 1 & 0.0000 \\
\hline 2 & 3 & 0 & 0.2333 & 0 & 0 & 0 & 0.0000 \\
\hline
\end{tabular}


Table B.2 - System signature for system European 3.

\begin{tabular}{|c|c|c|c|c|c|}
\hline $\begin{array}{l}\text { Number of } \\
\text { failed } \\
\text { components } \\
\text { (j) }\end{array}$ & $\begin{array}{l}\text { System } \\
\text { signature } \\
\text { value }\left(q_{j}\right)\end{array}$ & $\begin{array}{l}\text { Number of } \\
\text { failed } \\
\text { components } \\
\text { (j) }\end{array}$ & $\begin{array}{c}\text { System } \\
\text { signature } \\
\text { value }\left(q_{j}\right)\end{array}$ & $\begin{array}{l}\text { Number of } \\
\text { failed } \\
\text { components } \\
\text { (j) }\end{array}$ & $\begin{array}{c}\text { System } \\
\text { signature } \\
\text { value }\left(q_{j}\right)\end{array}$ \\
\hline 1 & $0.00 \mathrm{E}+00$ & 28 & 1.36E-09 & 55 & $9.41 \mathrm{E}-03$ \\
\hline 2 & $0.00 E+00$ & 29 & 3.55E-09 & 56 & $1.24 \mathrm{E}-02$ \\
\hline 3 & $0.00 \mathrm{E}+00$ & 30 & 8.81E-09 & 57 & $1.60 \mathrm{E}-02$ \\
\hline 4 & $0.00 E+00$ & 31 & $2.10 \mathrm{E}-08$ & 58 & $2.02 \mathrm{E}-02$ \\
\hline 5 & $0.00 \mathrm{E}+00$ & 32 & $4.80 \mathrm{E}-08$ & 59 & $2.49 \mathrm{E}-02$ \\
\hline 6 & $0.00 \mathrm{E}+00$ & 33 & $1.06 \mathrm{E}-07$ & 60 & $3.01 \mathrm{E}-02$ \\
\hline 7 & $0.00 E+00$ & 34 & 2.27E-07 & 61 & $3.56 \mathrm{E}-02$ \\
\hline 8 & $0.00 E+00$ & 35 & 4.72E-07 & 62 & 4.12E-02 \\
\hline 9 & $0.00 \mathrm{E}+00$ & 36 & $9.54 \mathrm{E}-07$ & 63 & 4.67E-02 \\
\hline 10 & $0.00 E+00$ & 37 & $1.88 \mathrm{E}-06$ & 64 & $5.18 \mathrm{E}-02$ \\
\hline 11 & $0.00 E+00$ & 38 & $3.60 \mathrm{E}-06$ & 65 & 5.63E-02 \\
\hline 12 & $0.00 E+00$ & 39 & 6.76E-06 & 66 & 5.99E-02 \\
\hline 13 & $0.00 E+00$ & 40 & $1.24 \mathrm{E}-05$ & 67 & $6.24 \mathrm{E}-02$ \\
\hline 14 & $0.00 \mathrm{E}+00$ & 41 & $2.22 \mathrm{E}-05$ & 68 & $6.37 \mathrm{E}-02$ \\
\hline 15 & $0.00 \mathrm{E}+00$ & 42 & $3.91 \mathrm{E}-05$ & 69 & 6.37E-02 \\
\hline 16 & $0.00 \mathrm{E}+00$ & 43 & $6.72 \mathrm{E}-05$ & 70 & $6.22 \mathrm{E}-02$ \\
\hline 17 & $0.00 E+00$ & 44 & $1.13 \mathrm{E}-04$ & 71 & 5.95E-02 \\
\hline 18 & $5.55 \mathrm{E}-16$ & 45 & $1.88 \mathrm{E}-04$ & 72 & $5.55 \mathrm{E}-02$ \\
\hline 19 & $5.66 \mathrm{E}-15$ & 46 & 3.04E-04 & 73 & $5.04 \mathrm{E}-02$ \\
\hline 20 & $4.09 \mathrm{E}-14$ & 47 & 4.83E-04 & 74 & 4.43E-02 \\
\hline 21 & $2.28 \mathrm{E}-13$ & 48 & 7.53E-04 & 75 & $3.74 \mathrm{E}-02$ \\
\hline 22 & $1.08 \mathrm{E}-12$ & 49 & $1.15 \mathrm{E}-03$ & 76 & $3.00 \mathrm{E}-02$ \\
\hline 23 & $4.44 \mathrm{E}-12$ & 50 & $1.72 \mathrm{E}-03$ & 77 & $2.23 \mathrm{E}-02$ \\
\hline 24 & $1.64 \mathrm{E}-11$ & 51 & $2.52 \mathrm{E}-03$ & 78 & $1.45 \mathrm{E}-02$ \\
\hline 25 & $5.51 \mathrm{E}-11$ & 52 & $3.62 \mathrm{E}-03$ & 79 & $6.96 \mathrm{E}-03$ \\
\hline 26 & $1.71 \mathrm{E}-10$ & 53 & $5.08 \mathrm{E}-03$ & 80 & $0.00 \mathrm{E}+00$ \\
\hline 27 & $4.98 \mathrm{E}-10$ & 54 & 6.99E-03 & & \\
\hline
\end{tabular}

